TITLE:

\title{
Fluctuation-response relation in a rocking ratchet
}

AUTHOR(S):

Harada, T; Yoshikawa, $\mathrm{K}$

CITATION:

Harada, $T$...[et al]. Fluctuation-response relation in a rocking ratchet. Physical Review E 2004, 69(3): 031113.

ISSUE DATE:

2004-03

URL:

http://hdl.handle.net/2433/49878

RIGHT:

Copyright 2004 American Physical Society 
PHYSICAL REVIEW E 69, 031113 (2004)

\title{
Fluctuation-response relation in a rocking ratchet
}

\author{
Takahiro Harada and Kenichi Yoshikawa* \\ Department of Physics, Graduate School of Science, Kyoto University \& CREST, Kyoto 606-8502, Japan \\ (Received 30 July 2003; published 31 March 2004)
}

\begin{abstract}
The relationship between the positional fluctuation and mechanical response for a thermal ratchet at a nonequilibrium steady state was investigated experimentally and considered theoretically. We constructed a ratchet system using a scanning optical trapping system and microbeads $(0.20 \mu \mathrm{m}$ diameter $)$, which is interpreted as a realization of a rocking ratchet [M. O. Magnasco, Phys. Rev. Lett. 71, 1477 (1993)]. In the experiment, an asymmetric periodic potential ( $2.5 \mu \mathrm{m}$ period) was generated by optical trap scanning, which traps a bead. When the potential profile was rocked sinusoidally, diffusion of the bead was rectified in one direction. We confirmed that both the diffusion coefficient and the mobility increased with potential rocking with a positive correlation. To obtain better insight, we performed numerical and theoretical analyses of the corresponding Langevin system. Although there is a positive relationship between the diffusion coefficient and mobility, the diffusion coefficient is greater than the value given in the Einstein relation. This result means that the effective temperature of the thermal ratchet at a nonequilibrium steady state becomes greater than that of the environment. We propose that this elevation of the effective temperature causes a decrease in the energetic efficiency of the thermal ratchet through irreversible dissipation to the heat bath.
\end{abstract}

DOI: 10.1103/PhysRevE.69.031113

PACS number(s): 05.40.Jc, 05.70.Ln, 42.62.-b, 87.16.Nn

\section{INTRODUCTION}

Recent experiments on biological molecular motors at a single-molecule level have revealed several curious aspects of their dynamic behavior, including their stochastic motion under a large noise [1], and excellent performance in chemomechanical energy transduction [2]. To understand these results, we must consider how thermodynamic considerations can be extended to molecular motors operating far from equilibrium, where the mechanism is completely different from that of the Carnot engine.

We investigated how concepts in equilibrium thermodynamics, such as temperature, appear in a highly nonequilibrium regime. An important part of thermodynamics is the fluctuation-dissipation theorem (FDT), which associates the fluctuation of an observable at equilibrium with its response to a small perturbation [3-5]. The Einstein relation [6] is an example; this theory states that the diffusion coefficient $D$ and the mobility $\mu$ of a colloidal particle suspended in aqueous solution at equilibrium have the following relation:

$$
D=\mu k_{\mathrm{B}} T,
$$

where $k_{\mathrm{B}}$ is Boltzmann constant and $T$ is the temperature of the system. Therefore, it seems important to investigate the relationship between the fluctuation and the response in molecular motors to address the above-mentioned problem.

In this paper, we present our experimental results concerning the relationship between the diffusion coefficient and mobility in a thermal ratchet, as a simple model of a molecular motor. Thermal ratchets, a class of theoretical models that rectify thermal and/or external noise into a net current of particles, have been intensively studied over the past decade $[7,8]$. There have been several studies on the average behav-

\footnotetext{
*Electronic address: yoshikaw@scphys.kyoto-u.ac.jp
}

ior of thermal ratchets, especially on the sign and magnitude of the induced probability current [9] and the energetic efficiency [10]. In contrast, there are few reports that focus on the fluctuation in the motion of ratchets, especially we are not aware of a study dealing with FDT on thermal ratchets.

Therefore, we examined the Einstein relation for a ratchet system, which is interpreted as a realization of a rocking ratchet $[11,12]$ (see the discussion in Sec. II A). A ratchet potential was experimentally constructed using scanning optical trapping and polystyrene microbeads $(0.20 \mu \mathrm{m}$ diameter). When a focused laser beam (optical tweezers) is scanned fast enough on the focal plane, a periodic asymmetric potential for the beads is generated at the orbit of the beam waist, upon which a trapped bead diffuses one dimensionally. When the periodic potential profile is sinusoidally rocked, a net current of the beads is generated. It is also found that the potential rocking increases both the diffusion coefficient and the mobility of the beads in a positive correlation.

Inspired by the above experimental results, we also performed the numerical and theoretical analyses on the corresponding Langevin equation. The numerical simulation showed that the present model reproduces the experimental trend qualitatively, including the positive correlation between the diffusion coefficient and mobility. However, a further analysis revealed that their relation deviates from the Einstein-type linear relation, and the ratio of the diffusion coefficient to mobility becomes greater than the temperature of the surrounding heat bath. Essentially the same result is also obtained from an analytical calculation for the case of a slow-rocking limit.

These results indicate that the effective temperature of the rocking ratchet in a nonequilibrium steady state, determined from the ratio of the diffusion coefficient to the mobility, is close to but greater than the temperature of the environment. We propose a simple formula to evaluate the energetic efficiency in relation to the effective temperature of the thermal 
ratchet, based on which the energetic efficiency of the present system was calculated. We suggest that the increase in the effective temperature causes dissipation between the particle and the surrounding heat bath, and decreases the energetic efficiency as an engine.

This paper is organized as follows. In Sec. II, we describe the experimental system and the results for the rocking ratchet. In Sec. III, we introduce the Langevin equation (and the Fokker-Planck equation) corresponding to the experimental setup, and show the results of the numerical simulation and the analytical calculation. In Sec. IV, we present our interpretation of the results and a consideration of the energetic efficiency. Our concluding remarks are given in Sec. V.

\section{EXPERIMENT}

In this section, we describe the experiment on an optical rocking ratchet. After explaining the sample preparation and optical setup, we describe the behavior of beads trapped on unrocked and rocked periodic potentials. We then describe the measurement of the mobility by applying a small external force, and then present the relationship between the measured mobility and the diffusion coefficient.

\section{A. Experimental setup}

We constructed an optical thermal ratchet by modifying the experimental system of Faucheux et al. [13]. The experimental setup is shown schematically in Fig. 1. Fluorescent polystyrene beads of $0.20 \mu \mathrm{m}$ in diameter (fluorspheres, molecular probes: size dispersion $\leqslant 5 \%$ ) were dispersed in pure water to a volume fraction of $2 \times 10^{-6}$. Double-sided adhesive tape (NW-15, Nichiban) was cut into a ring shape and placed as a spacer between microscope slides. The sample cell, filled with a suspension of the beads, was sealed with nail polish. The sample preparation was placed on an automatic stage (BIOS-201T, Sigma Koki) of a fluorescent microscope (TE-300, Nikon), and was observed with a SIT camera (Hamamatsu Photonics). The scanning optical trapping system is constructed as follows. A linearly polarized $\mathrm{TEM}_{00}$ beam with a wavelength of $1064 \mathrm{~nm}$ is output from a $\mathrm{Nd}^{3+}$ :YAG (yttrium aluminum garnet) laser (Millennia IR, Spectra Physics). The beam is deflected with an acoustooptic deflector (AOD, 2DS-50-30-1.06, Brimrose), after being expanded with two spherical lenses, and fed into a microscope, again via two lenses. The power of the incident beam was $0.80 \mathrm{~W}$. Upon strong focusing with an objective lens (Plan Fluo $\times 100$ oil immersion, numerical aperture of 1.30, Nikon), the beam forms an optical cone inside the sample cell, which generates an attractive potential well for the beads.

This optical system enables the beam waist to rapidly scan the focal plane of the objective lens. As described in Ref. [14], a microparticle in the optical field of a Gaussian beam waist, which is drawn sufficiently fast, feels a "continuous" potential field along the orbit of the beam waist. The beam waist was reciprocated along a straight line, where beads are confined with Brownian motion. The depth of the optical potential is proportional to the time-averaged inten-

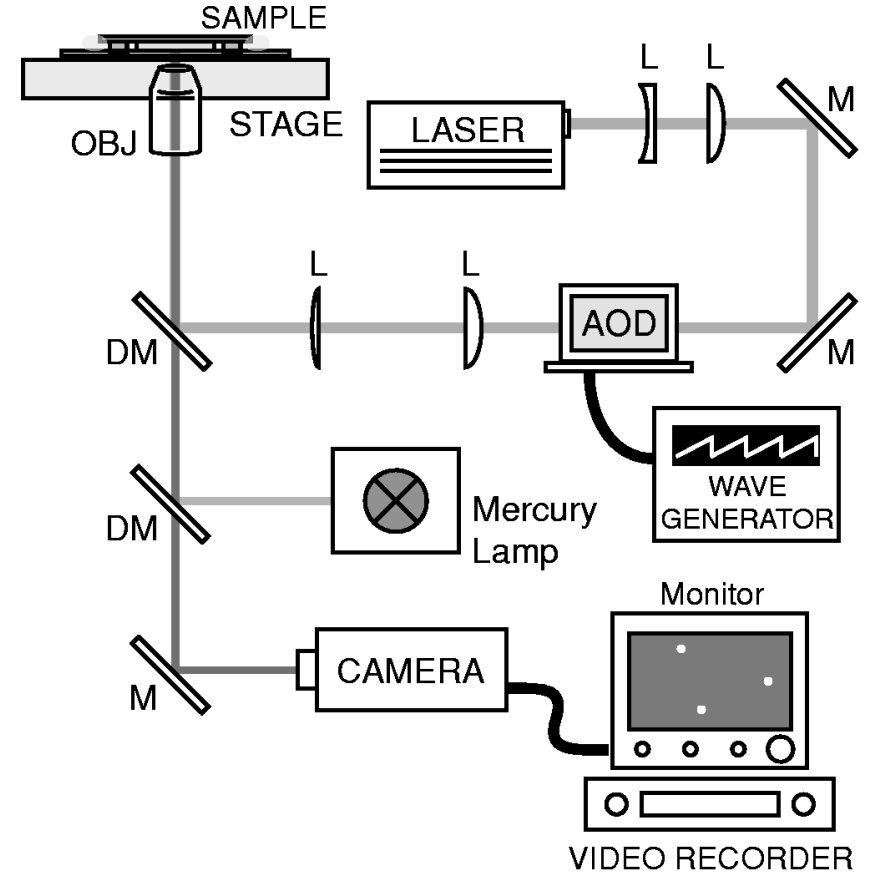

FIG. 1. Schematic of the experimental setup for an optical rocking ratchet. OBJ, DM's, M's, and L's indicate an objective lens, dichroic mirrors, mirrors, and lenses, respectively. A beam emitted from a Nd:YAG laser was expanded with a beam expander, deflected with an acousto-optic deflector (AOD), poured into the objective lens via relay lenses, and then focused. The fluorescent beads in the sample preparation were monitored with a SIT camera mounted on the fluorescent microscope, recorded and analyzed.

sity of the light [15], which is inversely proportional to the scanning velocity of the beam waist. We could thus construct a sawtoothed periodic potential profile by modulating the speed of the scanning beam waist. The beam waist was programmed to move according to $X(t)=L\left(\sqrt{1+8 t / t_{0}}-1\right) / 2$ for one period of the potential, where $L$ and $t_{0}$ are the length and duration of one period of the potential, respectively. The beam waist was drawn along a straight line, repeating the period of motion $X(t) 18$ times, then drawn back with reversed motion $X\left(t_{0}-t\right)$ for the same number of times, and the whole forward/backward scanning procedure was cycled. The length $L$ of a single period of the optical potential was set at $2.5 \mu \mathrm{m}$, and the scanning frequency, equal to $1 / 36 t_{0}$, was $300 \mathrm{~Hz}$. Figure 2 shows the potential profile for a single bead, evaluated as a logarithm of the probability distribution of a bead measured by tracing its motion within a single period [5]. The depth of the potential is estimated to be $5.5 k_{\mathrm{B}} T$.

We applied unbiased periodic forcing on the trapped beads by rocking the intensity profile of the light (Fig. 3). The whole intensity profile can be translated slowly along the direction of the scanning orbit. In this way, the intensity profile $I(x, t)$ was rocked in a sinusoidal manner as $I(x, t)$ $=I(x-A \sin \omega t)$. With the rocking, the bead trapped on the potential feels an additional viscous resistance from the solvent. If we adopt the coordinate system where the potential stays stationary, i.e., by transformation $x-A \sin \omega t \rightarrow x$, the bead looks being exerted a periodic force $F(t)$ 


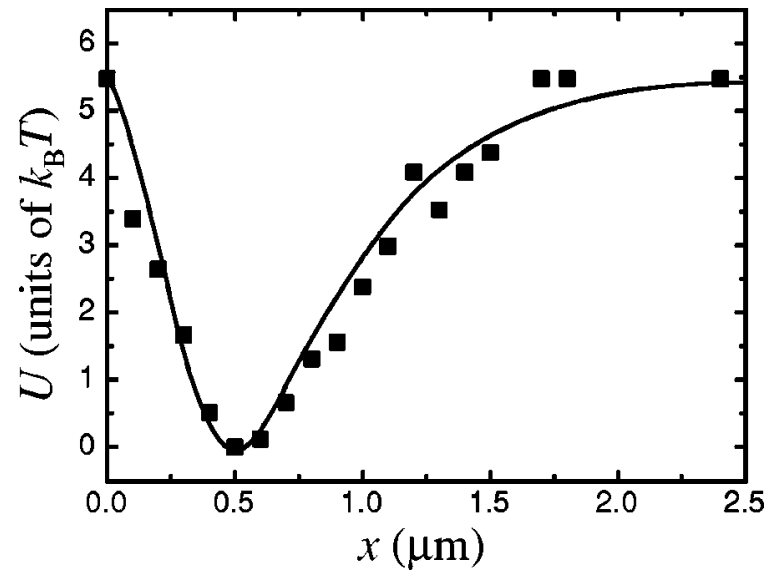

FIG. 2. Profile of the optically generated periodic potential for one period. The profile $U(x)$ is estimated from the probability distribution $P(x)$ of a $\phi=0.20 \mu \mathrm{m}$ bead, obtained by tracing its motion within one period of the potential, using the formula $U(x)$ $=-k_{\mathrm{B}} T \ln P(x)+G$, where $G$ is a constant [5].

$=-\gamma \omega A \cos \omega t$ by the solvent (see also Appendix for details). This kind of system, where a particle on a periodic potential is exerted a periodic force, is called a rocking ratchet [8]. Thus our system is considered as a realization of a rocking ratchet. In this study, the rocking frequency $\omega / 2 \pi$ was fixed at $10 \mathrm{~Hz}$, which is sufficiently slower than the scanning frequency of $300 \mathrm{~Hz}$, and the amplitude $A$ was varied from $0 L$ to $0.6 L$. In relation to the characteristics as a rocking ratchet, the amplitude of $F(t), F_{0}=\gamma \omega A$, is calculated as $0 \mathrm{fN}-1.8 \times 10^{2} \mathrm{fN}$ from these parameters. This amplitude corresponds to $0 k_{\mathrm{B}} T-1.2 \times 10^{2} k_{\mathrm{B}} T$ for a maximum potential drop per potential period, $F_{0} L$.

In the following experiments, a single particle was put on the optical potential for every trial. For each parameter, trials were repeated for around 100 times sequentially, and the data were analyzed statistically. All of the experiments were conducted at $21 \pm 1^{\circ} \mathrm{C}$.

\section{B. Experimental results}

In the experiment, stepwise movement of a single trapped bead over the periodic potential was observed. Figure 4 ex-

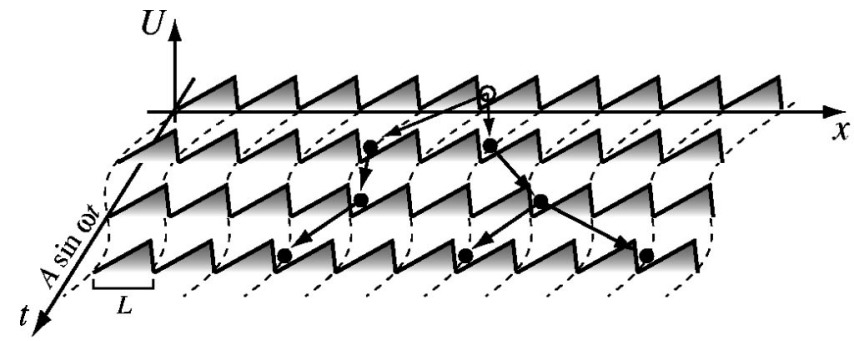

FIG. 3. Schematic of an optical rocking ratchet. An optically realized sawtoothed potential (period $L$ ) is rocked sinusoidally with amplitude $A$ and frequency $\omega$. A trapped microbead shows random and stepwise motion on the rocked periodic potential, where each period is of length $L$. Only a single bead is put on the potential for each trial, and trials are repeated.

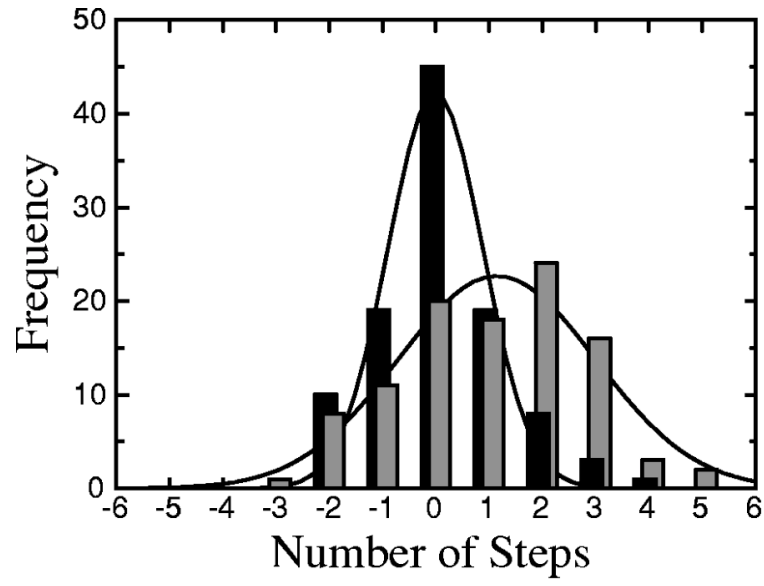

FIG. 4. Spatial distribution of stepwise movement of microbeads in an optical rocking ratchet. Histograms for displacement of beads initially placed at $x=0$ after $2 \mathrm{~s}$ of diffusion are shown in units of $L$. The black bars and the gray bars represent data for the stationary condition $(A=0 L)$ and one of the rocking conditions $(A=0.36 L)$, respectively. The solid lines are Gaussian fits.

emplifies the spatial distribution, in units of $L$, of beads initially placed at $x=0$ after $2 \mathrm{~s}$ of diffusion, under a stationary condition (i.e., $A=0 L$ ) and a rocking condition ( $A$ $=0.36 L)$. The probability densities under both conditions form Gaussian distributions. The center of the distribution in the rocking condition is shifted to the right (see Fig. 2), while that in the stationary condition is almost at zero. The width of the distribution in the rocking condition is greater than that in the stationary condition. In Fig. 5, the mean velocity $v$ of the beads is plotted as a function of the rocking amplitude. The mean velocity is determined from the average displacement of the beads in $2 \mathrm{~s}$. The graph shows that the velocity

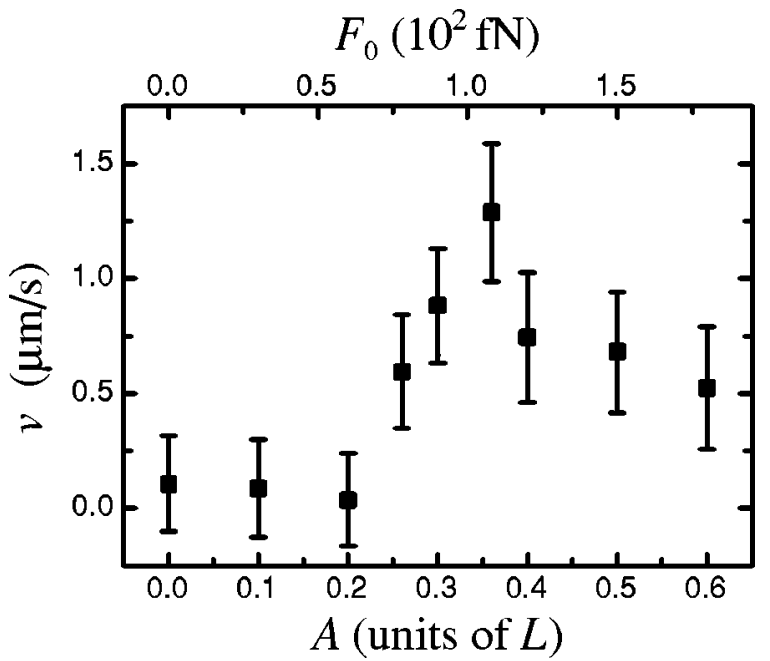

FIG. 5. Mean velocity $v$ of the beads against the amplitude $A$ of the potential rocking. The amplitude is given in units of the potential period $L$. The error bars are standard errors, which is also proportional to the magnitude of the diffusion coefficients. In the above axis, the magnitude of oscillating viscous force from solvent $F_{0}$ $=\gamma \omega A$ is also shown (see the argument in Sec. II A.). 


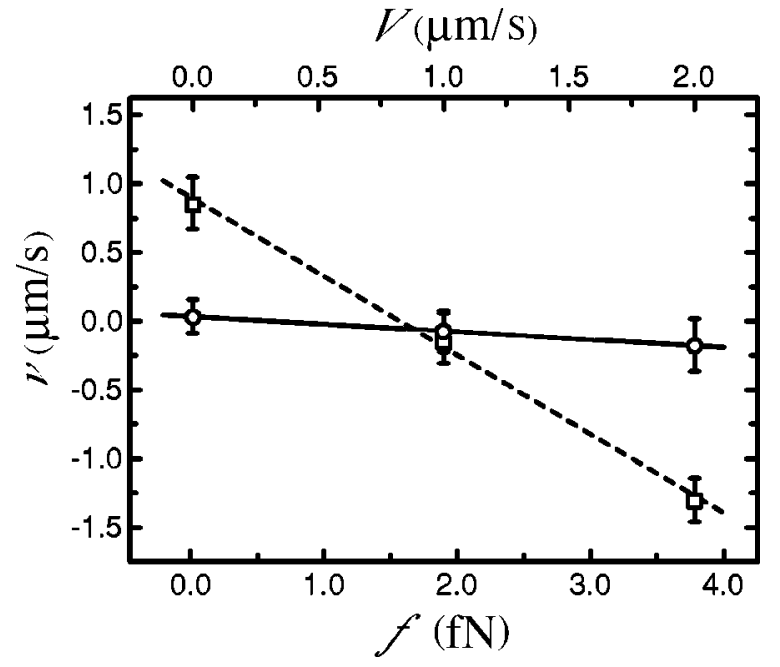

FIG. 6. Typical response of the mean velocity $v$ against the load force $f$. The open circles and squares correspond to the stationary condition $(A=0 L)$ and the rocking condition $(A=0.4 L)$, respectively. The lines are linear fits with $\chi^{2}$ fitting [26]. The force $f$ is calculated with Stokes' formula, from the translation velocity $V$ of the microscope stage [16].

remains almost zero when the rocking amplitude is small $(A \leqslant 0.2 L)$, and then increases with $A$ until it reaches a maximum at $A \simeq 0.35 L$.

Next, we examined the response of this ratchet system to an external load force. We used viscous resistance as a load force, which is exerted on the trapped beads by translating the microscope stage at a constant velocity. For translation velocity $V$, the load force is estimated to be $f=6 \pi \eta a \mathrm{~V}$, where $\eta=1.0 \times 10^{-6} \mathrm{~g} /(\mu \mathrm{ms})$ is the viscosity of the water and $a=0.10 \mu \mathrm{m}$ is the radius of the beads [16]. The direction of stage translation is set opposite the mean velocity of the beads. A typical response of the mean velocity to constant external force is given in Fig. 6 for the stationary and rocking conditions, where the mean velocity decreases linearly with the force. From the slope of the response line, we can evaluate the response coefficient (mobility) of the mean velocity to the external force. The mobility in the rocking condition is greater than that in the stationary condition.

In Fig. 7, the mobility is plotted against the diffusion coefficient without a load force for various rocking amplitudes. The diffusion coefficient is calculated from the second $\mathrm{cu}-$ mulant of the step distribution in $2 \mathrm{~s}$ (see, e.g., Fig. 4), i.e., $D=L^{2}\left\langle(x-\langle x\rangle)^{2}\right\rangle / 2 t$, where $L=2.5 \mu \mathrm{m}$ and $t=2 \mathrm{~s}$. The data points from the bottom to the top in the graph correspond to the cases of $A / L=0,0.20,0.26,0.40$, respectively. The mobility increases with an increase in the rocking amplitude, as well as the diffusion coefficient. The extrapolation of the plot, however, does not go through the origin, as shown in the inset for Fig. 7. According to the graph, the data plot seems to have a certain offset from the Einstein relation.

\section{THEORY}

Since the statistical error in Fig. 7 is so large, we cannot determine whether the relation between the diffusion coeffi-

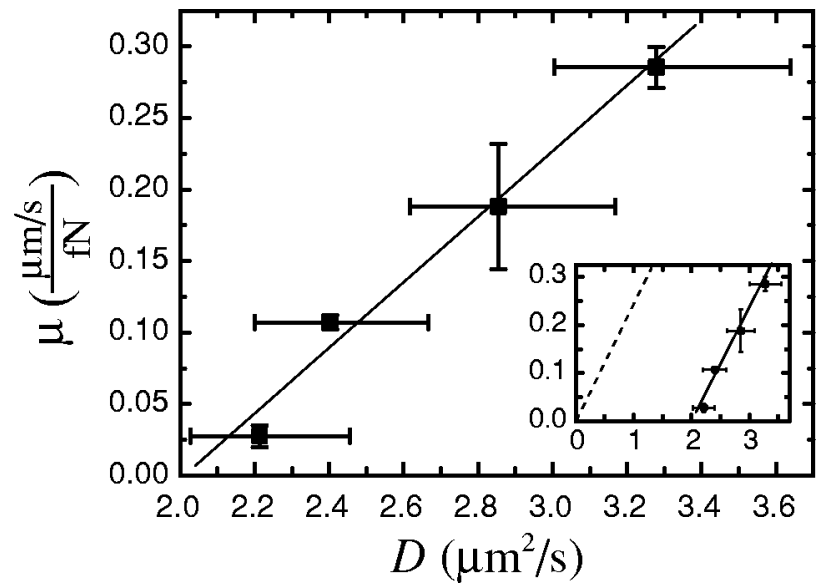

FIG. 7. Relationship between the diffusion coefficient $D$ and mobility $\mu$. The diffusion coefficient without a viscous load and the mobility against a load are plotted for different values of the rocking amplitude, $0 L, 0.20 L, 0.26 L, 0.40 L$, from the bottom to the top. The error bars for $D$ represent $50 \%$ confidence intervals in $\chi^{2}$ distribution [27]. The error bars for $\mu$ were calculated by $\chi^{2}$ fitting on the force-velocity relation (see Fig. 6). The solid line is the linear fit whose slope is $4.2 \times 10^{-21} \pm 0.48 \times 10^{-21} \mathrm{~J}\left(=3.0 \times 10^{2} \pm 3.4\right.$ $\left.\times 10^{1} \mathrm{~K}\right)$. Inset: The same data displayed with the Einstein relation (dashed line).

cient and the mobility is linear or not only from the experiment. Thus we examined the corresponding mathematical model to obtain a deeper insight. In this section, we introduce a mathematical model of an overdamped Langevin equation, along with the results of a numerical simulation which show a qualitative correspondence to the experimental trend. An analytical calculation in the limiting case of slow rocking is also described.

\section{A. Model}

Since the relaxation time of the beads $(\mathrm{m} / \gamma=2.2$ $\times 10^{-9} \mathrm{~s}$ ) is negligible compared to the time scale of their stepping motion (subseconds) in the experiment, the motion of a microparticle can be described with an overdamped Langevin equation of the form

$$
\begin{gathered}
\gamma \dot{x}=-\partial_{x} W(x, t)+f+\xi(t), \\
\langle\xi(t)\rangle=0,\left\langle\xi(t) \xi\left(t^{\prime}\right)\right\rangle=2 \gamma k_{\mathrm{B}} T \delta\left(t-t^{\prime}\right),
\end{gathered}
$$

or the equivalent Fokker-Planck equation

$$
\partial_{t} P(x, t)=-\partial_{x}\left(-\partial_{x} W(x, t)+f-k_{\mathrm{B}} T \partial_{x}\right) P(x, t) / \gamma
$$

$\gamma, W(x, t), f, \xi(t)$, and $k_{\mathrm{B}} T$ represent the viscous coefficient of the solvent, the rocked optical potential, the constant external force, the thermal noise, and the temperature of the surrounding solvent, respectively [5,17]. For simplicity, the profile of the periodic optical potential, shown in Fig. 3, is approximated with a piecewise-linear function 


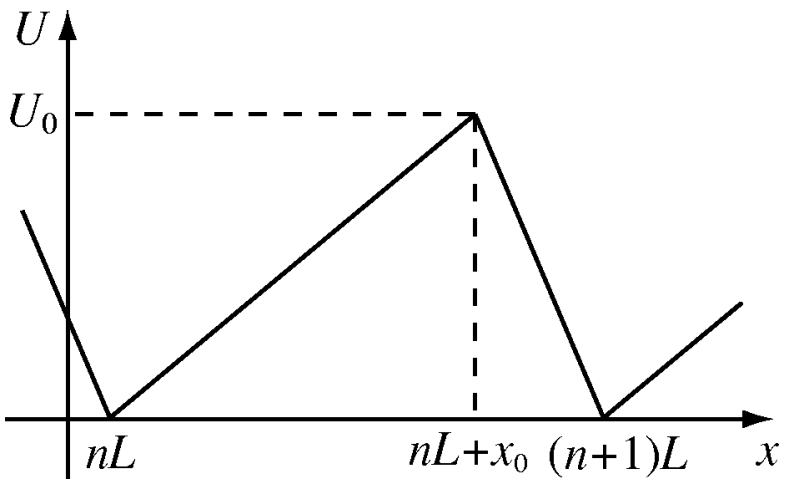

FIG. 8. Piecewise-linear potential used in the mathematical model [Eq. (4)]. $U_{0}$ and $x_{0}$ are positive constants, $L$ is the period of the potential, and $n$ is an integer. In the present paper, we have adopted the values $U_{0}=5 k_{\mathrm{B}} T$ and $x_{0}=0.8 \mathrm{~L}$.

$$
\begin{aligned}
& U(x)= \begin{cases}\frac{U_{0}}{x_{0}}(x-n L) & \left(n L \leqslant x<n L+x_{0}\right) \\
-\frac{U_{0}}{1-x_{0}}[x-(n+1) L] & {\left[n L+x_{0} \leqslant x<(n+1) L\right]}\end{cases} \\
&(n: \text { integer }),
\end{aligned}
$$

where $U_{0}$ and $x_{0}(<L)$ are positive constants and $L$ is the period of the potential (see Fig. 8). Since the intensity profile of the light is rocked sinusoidally, the optical potential depends on time as $W(x, t)=U(x-A \sin \omega t)$.

In connection to the experimental parameters, $L, k_{\mathrm{B}} T$, and $\gamma$ correspond to $2.5 \mu \mathrm{m}, 4.1 \times 10^{-21} \mathrm{~J}$, and $1.9 \times 10^{-9} \mathrm{~kg} / \mathrm{s}$, respectively. Thus the unit of time becomes $\tau \equiv L^{2} \gamma / k_{\mathrm{B}} T$ $=2.9 \mathrm{~s}$. In this notation, the other experimental parameters were $U_{0} \approx 5.5 k_{\mathrm{B}} T, x_{0} \approx 0.8 L, A=0 L-0.6 L$, and $\omega / 2 \pi$ $=10 \mathrm{~Hz} \approx 30 \tau^{-1}$. On the other hand, the parameters in the mathematical model were chosen as $U_{0}=5 k_{\mathrm{B}} T, x_{0}=0.8 \mathrm{~L}$, $A=0 L-0.6 L$, and $\omega / 2 \pi=15 \tau^{-1}$ for the following simulation and calculation.

\section{B. Numerical results}

In Fig. 9(a), the mean velocity in the numerical simulation is plotted against the rocking amplitude. The graph corresponds well to the profile of the velocity-amplitude relation obtained in the experiment (see Fig. 5). We then investigated the relation between the diffusion coefficient and mobility. Figure 9 shows the numerical result for this relationship at every value of the rocking amplitude. A positive, almost linear, correlation between the diffusion coefficient and mobility is also found in the numerical simulation. However this relationship deviates from the Einstein-type linear relation. In the numerical simulation, the diffusion coefficient and mobility at $A=0 L$, namely, the stationary condition, satisfy the Einstein relation, unlike the experiment. This is because the stationary condition in this model exactly describes a simple Brownian motion in a static potential, which is considered to be in equilibrium.
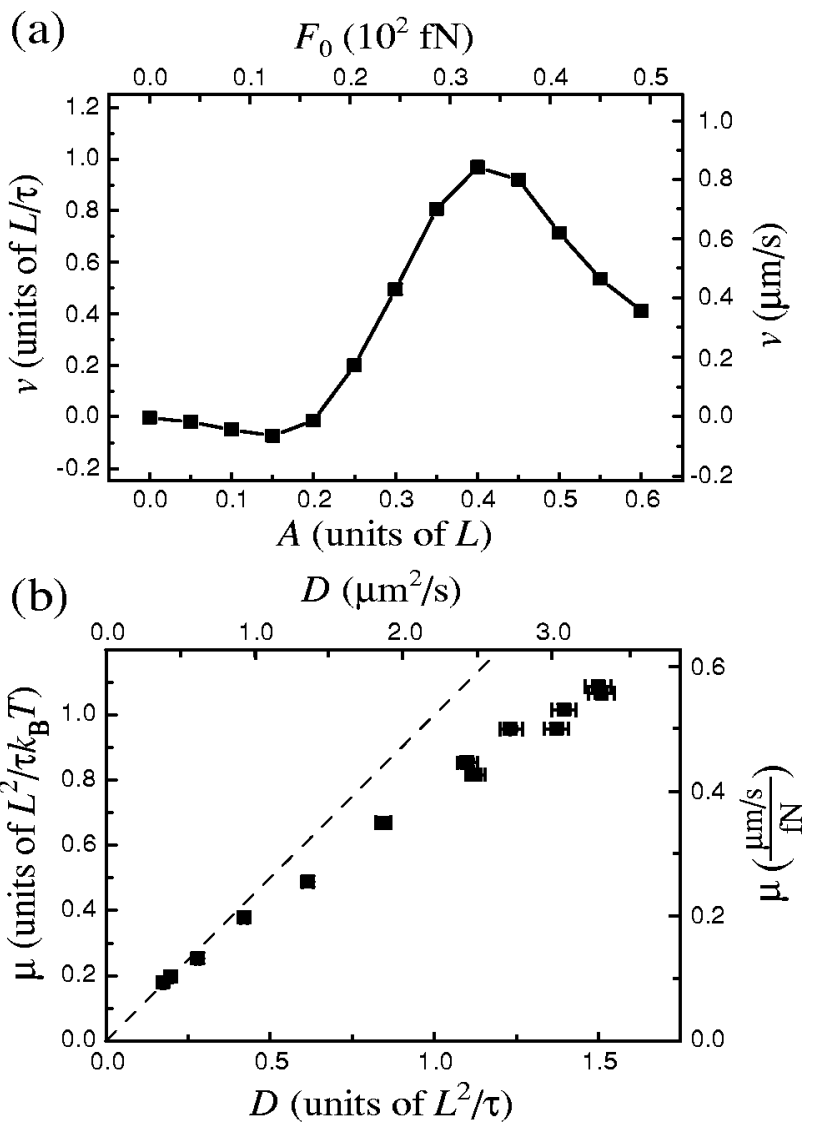

FIG. 9. Results of numerical simulation. (a) Mean velocity $v$ against the rocking amplitude $A$. In the left and right axes, $v$ is represented in units of $L / \tau$ and $\mu \mathrm{m} / \mathrm{s}$, respectively. $A$ is represented in units of $L$ at the bottom with corresponding $F_{0}=\gamma \omega A$ at the top. (b) Relationship between the diffusion coefficient $D$ and mobility $\mu$ for the mathematical model of Eqs. (2) and (4). The diffusion coefficient $D$ (at the bottom in units of $L^{2} / \tau$ and at the top in units of $\mu \mathrm{m}^{2} / \mathrm{s}$ ) under no external force and the mobility $\mu$ [at the left in units of $L^{2} /\left(\tau k_{\mathrm{B}} T\right)$, and at the right in units of $\left.\mu \mathrm{m} /(\mathrm{s} \mathrm{fN})\right]$ against an external force are plotted for various values of the rocking amplitude from $0 L$ to $0.6 L$. The dashed line indicates the Einstein relation $D=\mu k_{\mathrm{B}} T$.

\section{Analytical solution}

In addition to the numerical study, we performed an analytical calculation of various observables for the present model, in the limiting case of $\omega \rightarrow 0$. The details of the calculation are presented in the Appendix. The results of this analytical investigation are shown in Fig. 10 for both the mean velocity and the relation between the diffusion coefficient and mobility, both of which show qualitative correspondence to the numerical results for finite $\omega$. In the case of small $A / L$, the analytical solution leads to an asymptotic expression as

$$
D(A)-D_{0}=\frac{D_{\mathrm{s}}^{(2)}(0)}{v_{\mathrm{s}}^{(3)}(0)}\left[\mu(A)-\mu_{0}\right]
$$

where $D(A)$ and $D_{0}$ are the diffusion coefficients for the rocking and stationary conditions, respectively, and $\mu(A)$ 


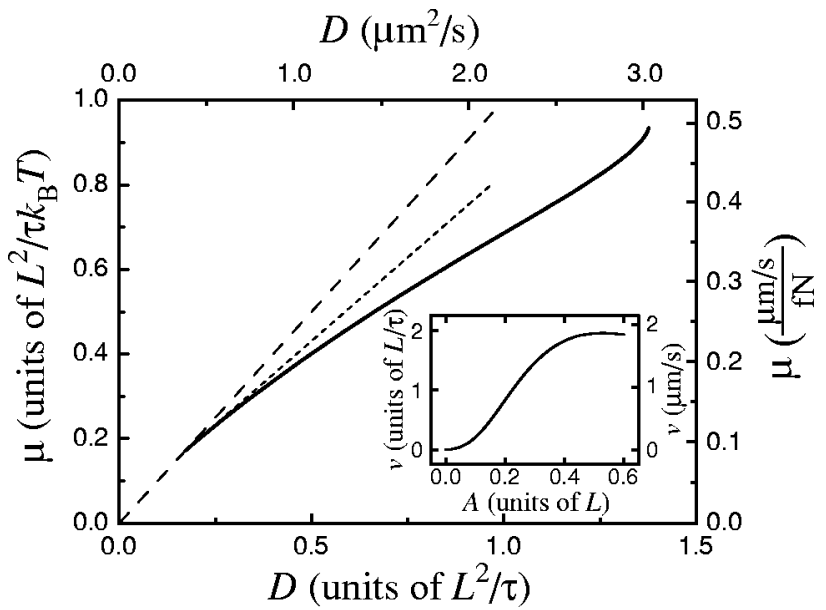

FIG. 10. Analytical result for the relationship between the diffusion coefficient $D$ and mobility $\mu$ for the model of Eqs. (2) and (4) with the limit $\omega \rightarrow 0$. The units are the same as in Fig. 9(b). The diffusion coefficient and the mobility are plotted for values of the rocking amplitude from $0 L$ to $0.6 L$. The dotted line is an asymptotic fit in the limit $A \rightarrow 0$ with a slope of $1.27 k_{\mathrm{B}} T$ [see Eq. (5)]. The dashed line indicates the Einstein relation. Inset: the analytical result for the mean velocity $v$ against the rocking amplitude $A$. The units are the same as in Fig. 9(a).

and $\mu_{0}$ are the mobility for the rocking and stationary conditions. In this case, the Einstein relation also holds for the stationary condition as $D_{0}=\mu_{0} k_{\mathrm{B}} T . v_{\mathrm{s}}(f)$ and $D_{\mathrm{s}}(f)$ are the velocity and the diffusion coefficient over a stationary periodic potential with constant external force $f$ (see the Appendix for details). The coefficient $D_{\mathrm{s}}^{(2)}(0) / v_{\mathrm{s}}^{(3)}(0)$ is equal to $1.27 k_{\mathrm{B}} T$ for the parameters used here, and is greater than $k_{\mathrm{B}} T$.

\section{DISCUSSION}

In this section, we discuss the physical meaning of the results. First, before discussing the connection to the FDT, we discuss the interpretation of the experimental results, especially for the stationary condition. Next, we define the effective temperature of the rocking ratchet in a nonequilibrium steady state in connection with the Einstein relation. Finally, we discuss the physical meaning of the effective temperature in terms of the energetic efficiency of the thermal ratchet.

\section{A. Connection to the Einstein relation}

In the experiment, although we found a positive correlation between the diffusion coefficient and mobility, the whole data points in the inset in Fig. 7 do not obey the Einstein relation. Especially, even the stationary condition does not satisfy the Einstein relation $(A=0 L$ : the lowest data point in the inset for Fig. 7). This significant deviation from the Einstein relation in the stationary condition occurs because the optical potential was generated by scanning of the beam waist, and was not static. In the stationary condition, although it is naively expected that a periodic potential is formed adiabatically since scanning of the optical potential well was sufficiently fast $(300 \mathrm{~Hz})$, the experimental results show that such an approximation is not correct [18]. Such an amplification on the diffusion coefficient has also been previously observed by Faucheux et al. for a Brownian particle diffusing in a circular track generated with scanning optical tweezers (see Fig. 11 in Ref. [14]). On the other hand, the additive increase in the diffusion coefficient in the rocking condition is due to slower rocking $(10 \mathrm{~Hz})$. Thus, separation of the time scales may enable us to divide the diffusion coefficient into several components as

$$
D(A)=D_{\text {eq }}+D_{\text {fast }}+D_{\text {slow }}(A),
$$

where $D_{\text {eq }}, D_{\text {fast }}$, and $D_{\text {slow }}$ are the diffusion coefficient which satisfies the Einstein relation and the components of the increment due to fast scanning and slow rocking, respectively. In this interpretation, $D_{\text {eq }}+D_{\text {fast }}$ is equal to the diffusion coefficient at $A=0 L, D(0 L)=2.2 \pm 0.18 \mu \mathrm{m}^{2} / \mathrm{s}$. On the other hand, $D_{\text {slow }}(0.4 L)=(3.3 \pm 0.27)-\left(D_{\text {eq }}+D_{\text {fast }}\right)$ $=1.1 \pm 0.46 \mu \mathrm{m}^{2} / \mathrm{s}$, for example. From this consideration, it is apparent that the stationary condition in the experiment does not correspond to thermal equilibrium. On the other hand, in the mathematical model, Eqs. (2) and (4), the Einstein relation holds in the stationary condition, and this case can be regarded as under equilibrium (i.e., $D_{\text {fast }}=0$ ) [19].

Thus, the experimental results can be summarized as showing that the diffusion coefficient and the mobility of a rocking ratchet increase in a positive correlation, with an increase in the rocking amplitude $A$. Furthermore, the mathematical model predicts that the Einstein relation does not hold in the nonequilibrium steady state. The ratio of the diffusion coefficient to mobility can be defined as the effective temperature of the thermal ratchet,

$$
k_{\mathrm{B}} T_{\mathrm{eff}}(A) \equiv \frac{D(A)}{\mu(A)},
$$

which in our model matches the temperature of the surrounding heat bath in equilibrium. In a nonequilibrium steady state, however, the effective temperature is greater than that of the heat bath. Such a nonequilibrium effect on a thermal ratchet may be attributed to the breaking of a detailed balance and seems to be rather general, independent of the detailed mechanism of the ratchet, including a flashing ratchet [20], and a correlation ratchet [21] (data not shown).

\section{B. Energetics}

We now address the energetics for the ratchet system in terms of the effective temperature. Recently an expression for the energetic efficiency of a thermal ratchet has been suggested using an effective temperature, which is essentially identical to $T_{\text {eff }}$ introduced here, by considering a large scale description of the motion of a particle [22]. According to the theory, the energetic efficiency of a thermal ratchet is expressed as

$$
e=\frac{f v}{2 \kappa k_{\mathrm{B}} \Delta T+F v},
$$




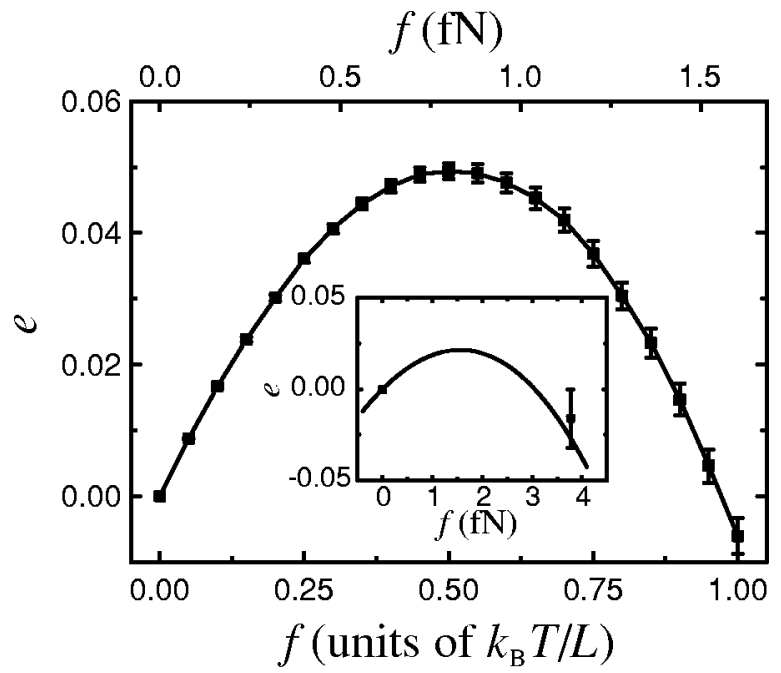

FIG. 11. Energetic efficiency $e$ against an external load $f$ (at the bottom in units of $k_{\mathrm{B}} T / L$ and at the top in units of $\mathrm{fN}$ ), numerically calculated using Eq. (8) for the present mathematical model $(A$ $=0.40 L)$. The time constant $\kappa^{-1}=0.186 \tau=0.54 \mathrm{~s}$ is adopted from the numerically determined mean first passage time, which is independent of the load force $f$. Inset: Experimentally determined energetic efficiency $e$ against an external load $f$ in the rocking condition $(A=0.40 L)$. The solid line is the calculated curve with the following values: the effective force $F=3.1 \mathrm{fN}$, the mobility $\mu=0.29 \mu \mathrm{m} /$ $(\mathrm{s} \mathrm{fN})$, time constant (mean first passage time) $\kappa^{-1}=0.57 \mathrm{~s}$, and effective temperature rise $k_{\mathrm{B}} \Delta T=8.5 \times 10^{-21} \mathrm{~J}$.

where $v$ is the mean velocity, $F \equiv v_{0} / \mu\left(v_{0}\right.$ is the mean velocity under no load) is the effective drifting force of the thermal ratchet, $f$ is the external load, $\Delta T \equiv T_{\text {eff }}-T$ is the effective temperature rise, and $\kappa^{-1}$ is the time constant which has a length close to the mean first passage time for the periodic potential. The mean velocity $v$ obeys the relationship $v=\mu(F-f)$.

Since the denominator of Eq. (8) contains $\kappa \Delta T$, a higher effective temperature decreases the energetic efficiency. When $\Delta T>0$, dissipation occurs even without a net current of the particle. Therefore, the efficiency reaches 0 in the quasistatic case $(v \rightarrow 0)$, while a finite velocity results in higher efficiency. Figure 11 shows the energetic efficiency $e$ as a function of the external load $f$, indicating a maximum at a finite velocity. On the other hand, Eq. (8) becomes unity in the ideal case of $\Delta T \rightarrow 0$ and $v \rightarrow 0$, i.e., in a reversible process. In other words, this expression of efficiency means "how much work can be extracted from thermodynamically available free energy?" [23]. Therefore, it must be distinguished from the usual concept of thermodynamic efficiency (e.g., for a Carnot engine).

\section{CONCLUSION}

In summary, we constructed a micrometer-sized rocking ratchet system using a scanning optical trap and microbeads. It is clear that the diffusion coefficient and the mobility are amplified in this operating thermal ratchet under a nonequilibrium steady state, and they show a positive and monotonic correlation. For the case of a corresponding mathematical model, we found that their relation deviates from an Einstein-type linear relation, indicating a larger diffusion coefficient. These results support the notation that the effective temperature for a rocking ratchet operating far from equilibrium is greater than that at equilibrium.

It may be a challenging problem to associate the effective temperature with the magnitude of violation of the detailed balance. It would also be interesting to conduct experiments on biological molecular motors from this perspective. Recent techniques involving single-molecule measurement would be the most suitable for such a subject [24]. This could be a new benchmark for testing theoretical models against real molecular motors.

\section{ACKNOWLEDGMENTS}

We thank Dr. S. Sasa and K. Hayashi for their focused discussions on this subject. We also thank Dr. K. Kitahara and Dr. J. Gorecki for their helpful advice. This work was supported in part by the Japan Society for the Promotion of Science for Young Scientists (Grant No. 05494).

\section{APPENDIX}

We describe in detail the calculation of various observables, such as the velocity, diffusion coefficient, and mobility, in the case of a slow-rocking limit. First, we perform a transformation $x-A \sin \omega t \rightarrow y$, on Eq. (2) to get the equations

$$
\begin{gathered}
\gamma \dot{y}=-\partial_{y} U(y)-\gamma A \omega \cos \omega t+f+\xi(t), \\
\langle\xi(t)\rangle=0,\left\langle\xi(t) \xi\left(t^{\prime}\right)\right\rangle=2 \gamma k_{\mathrm{B}} T \delta\left(t-t^{\prime}\right),
\end{gathered}
$$

where the notations are the same as those in Eq. (2). This Langevin equation corresponds to one known as a rocking ratchet. Since the momenta for $x\left(\langle x\rangle,\left\langle x^{2}\right\rangle\right.$, and so on) match those for $y$ with a long-time limit, it is sufficient to calculate the velocity, diffusion coefficient, and mobility for $y$.

Next, we consider a system with only a constant external force instead of periodic forcing as follows:

$$
\begin{gathered}
\gamma \dot{y}=-\partial_{y} U(y)+f+\xi(t), \\
\langle\xi(t)\rangle=0,\left\langle\xi(t) \xi\left(t^{\prime}\right)\right\rangle=2 \gamma k_{\mathrm{B}} T \delta\left(t-t^{\prime}\right) .
\end{gathered}
$$

For a periodic potential $U(y)$ with a period $L$, the velocity and diffusion coefficient can be given as [25]

$$
v_{\mathrm{s}}(f)=\frac{1-\exp \left(-f L / k_{\mathrm{B}} T\right)}{\int_{0}^{L} \frac{d y}{L} I_{+}(y)},
$$

$$
D_{\mathrm{s}}(f)=\frac{k_{\mathrm{B}} T}{\gamma} \frac{\int_{0}^{L} \frac{d y}{L} I_{+}(y)^{2} I_{-}(y)}{\left[\int_{0}^{L} \frac{d y}{L} I_{+}(y)\right]^{3}},
$$


where

$$
I_{ \pm}(y)=\frac{\gamma}{k_{\mathrm{B}} T} \int_{0}^{L} \frac{d z}{L} \exp \left\{( \pm U(y) \mp U(y \mp z)-z f) / k_{\mathrm{B}} T\right\} .
$$

We next proceed to the case of the periodic forcing. In the limit of $\omega \longrightarrow 0$, the velocity and diffusion coefficient are asymptotically calculated as

$$
\begin{aligned}
& v(A)=\frac{\omega}{2 \pi} \int_{0}^{2 \pi / \omega} d t v_{\mathrm{s}}(-\gamma A \omega \cos \omega t), \\
& D(A)=\frac{\omega}{2 \pi} \int_{0}^{2 \pi / \omega} d t D_{\mathrm{s}}(-\gamma A \omega \cos \omega t),
\end{aligned}
$$

where $2 \pi / \omega$ must be sufficiently greater than the relaxation time of the system of Eq. (A2). Similarly, the mobility is given as

$$
\mu(A)=\lim _{f \rightarrow 0} \frac{\omega}{2 \pi} \frac{\partial}{\partial f} \int_{0}^{2 \pi / \omega} d t v_{\mathrm{s}}(-\gamma A \omega \cos \omega t+f) .
$$

Since the periodic potential $U(y)$ is given in Eq. (4), the velocity, diffusion coefficient, and mobility can be calculated using this procedure. The results of the calculation are shown in Fig. 10.
[1] K. Svoboda, C.F. Schmidt, B.J. Schnapp, and S.M. Block, Nature (London) 365, 721 (1993).

[2] H. Tanaka et al., Nature (London) 415, 192 (2002).

[3] R. Graham and H. Haken, Z. Phys. 243, 289 (1971).

[4] G.S. Agarwal, Z. Phys. 252, 25 (1972).

[5] H. Risken, The Fokker-Planck Equation: Methods of Solution and Applications (Springer-Verlag, Berlin, 1989).

[6] A. Einstein, in Investigations on the Theory of the Brownian Motion, edited by R. Fürth, translated by A. D. Cowper (Dover, New York, 1956).

[7] F. Jülicher, A. Ajdari, and J. Prost, Rev. Mod. Phys. 69, 1269 (1997).

[8] P. Reimann, Phys. Rep. 361, 57 (2002).

[9] P. Reimann and P. Hänggi, Appl. Phys. A: Solids Surf. 75, 169 (2002).

[10] J.M.R. Parrondo and B.J. De Cisneros, Appl. Phys. A: Solids Surf. 75, 179 (2002).

[11] M.O. Magnasco, Phys. Rev. Lett. 71, 1477 (1993).

[12] R. Bartussek, P. Hänggi, and J.G. Kissner, Europhys. Lett. 28, 459 (1994).

[13] L.P. Faucheux, L.S. Bourdieu, P.D. Kaplan, and A.J. Libchaber, Phys. Rev. Lett. 74, 1504 (1995).

[14] L.P. Faucheux, G. Stolovitzky, and A. Libchaber, Phys. Rev. E 51, 5239 (1995).
[15] T. Tlusty, A. Meller, and R. Bar-Ziv, Phys. Rev. Lett. 81, 1738 (1998).

[16] See, for example, L.D. Landau and E.M. Lifshitz, Fluid Mechanics (Pergamon Press, London, 1987).

[17] A.S. Mikhailov and A.Yu. Loskutov, Chaos and NoiseFoundations of Synergetics II (Springer-Verlag, Berlin, 1996).

[18] The reason for and the mechanism of the FDT violation under breaking of the adiabatic approximation are being considered K. Hayashi, T. Harada, and S. Sasa (unpublished).

[19] K. Tomita and H. Tomita, Prog. Theor. Phys. 51, 1731 (1974).

[20] R.D. Astumian and M. Bier, Phys. Rev. Lett. 72, 1766 (1994).

[21] C.R. Doering, W. Horsthemke, and J. Riordan, Phys. Rev. Lett. 72, 2984 (1994).

[22] T. Harada (unpublished); e-print cond-mat/0310547.

[23] This type of definition of efficiency is also discussed in the following: I. Derényi, M. Bier, and R.D. Astumian, Phys. Rev. Lett. 83, 903 (1999).

[24] K. Svoboda, P.P. Mitra, and S.M. Block, Proc. Natl. Acad. Sci. U.S.A. 91, 11782 (1994).

[25] P. Reimann et al., Phys. Rev. Lett. 87, 010602 (2001).

[26] P.R. Bevington, Data Reduction and Error Analysis for the Physical Sciences (McGraw-Hill, New York, 1969).

[27] G.W. Snedecor and W.G. Cochran, Statistical Methods (Iowa State University Press, Iowa, 1989). 